\title{
ABSORPTION BY NEUTRAL HYDROGEN IN \\ THE IRREGULAR GALAXY M82
}

\author{
M. GUÉLIN \\ Observatoire de Meudon, 92-Meudon, France \\ and \\ L. WELIACHEW \\ California Institute of Technology, Pasadena, Calif. U.S.A.
}

(To be published in Astron. Astrophys.)

\begin{abstract}
A neutral hydrogen survey of the irregular galaxy M82 has been carried out with the transit radio telescope at Nançay, France. The resolving power was $4^{\prime}$ in right ascension and $34^{\prime}$ in declination. The velocity resolution was $59 \mathrm{~km} \mathrm{~s}^{-1}$.

Drift scans covering $58^{\prime}$ in right ascension were taken across the center of the galaxy where the radio source is located; fourteen scans were averaged.

Line profiles were derived every $2^{\prime}$ in right ascension. The profiles at $\pm 4^{\prime}$ from the radio source are similar within the measurement errors. In particular, they do not show any rotation effect within $\pm 15 \mathrm{~km} \mathrm{~s}^{-1}$. They were averaged in order to provide an estimate of the expected emission profile at the radio source position.

Subtraction of this average from the line profile measured in front of the radio source yielded significant negative temperatures at all velocities from 180 to $360 \mathrm{~km} \mathrm{~s}^{-1}$ and no positive temperatures at other velocities.

These negative temperatures were assigned to absorption of the radiation from the radio source by neutral hydrogen in $\mathbf{M} 82$.

Absorption is running from 3 to $6 \%$ in depth. The average velocity of the absorption profile is lying between the central emission velocity and the optically-determined velocity which are known to show a large disagreement.

The width of the absorption profile shows a velocity gradient of $200 \mathrm{~km} \mathrm{~s}^{-1}$ across the $35^{\prime \prime} \times 20^{\prime \prime}$ radio source. Such a large velocity spread across this angular extent is only shown by the excited gas showing emission lines at optical wavelengths (Burbidge et al., 1964).

Since then, the absorption in M82 at the neutral hydrogen wavelength has been confirmed by measurements done with the Owens Valley Radio interferometer at 2 spacings where any emission is completely resolved (interfringes were $3^{\prime}$ and $1^{\prime} .5$ ).

In addition to the conclusions of the single dish observations done at Nançay, the interferometer data have shown that the steeper velocity gradient occurs along the major axis of the galaxy which coincides with the major axis of the radio source.
\end{abstract}

\section{Reference}

Burbidge, E. M., Burbidge, G. R., and Rubin, V. C.: 1964, Astrophys. J. 140, 942. 